Chapter 5

Micro-Assembly Applications 


\title{
INTEREST OF THE INERTIAL TOLERANCING METHOD IN THE CASE OF WATCH MAKING MICRO ASSEMBLY
}

\author{
Maurice Pillet, Dimitri Denimal, Pierre-Antoine Adragna, Serge Samper \\ SYMME Laboratory of Université de Savoie, \\ B.P.806, 74016 Annecy, cedex \\ maurice.pillet@univ-savoie.fr, dimitri.denimal@univ-savoie.fr, \\ pierre-antoine.adragna@univ-savoie.fr, serge.samper@univ-savoie.fr,
}

Abstract A mechanical part to tolerance is traditionally expressed as a [Min Max] interval which allows the definition of the conformity of the characteristic. Inertial tolerancing offers a new point of view of the conformity based on the mean square deviation to the target. This article demonstrates the efficiency of inertial tolerancing and proposes a comparison with the traditional tolerancing method in the case of watch making micro assembly.

\section{Introduction}

The functional quality of a mechanical assembly is often a direct function of a functional clearance. In assemblies of watch making, this clearance results from the combination of several elementary components. In micro-assembly, tolerances are very tight with regard to the dimensions, and the yields of these assemblies are often weak. Moreover, in the case of the assembly of a bridge on the main plate, the same assembly must satisfy several clearances (Fig 1). The statistical combination of several clearances having a weak yield leads to an extremely weak global assembly yield. That results in numerous and expensive final improvements. [1]

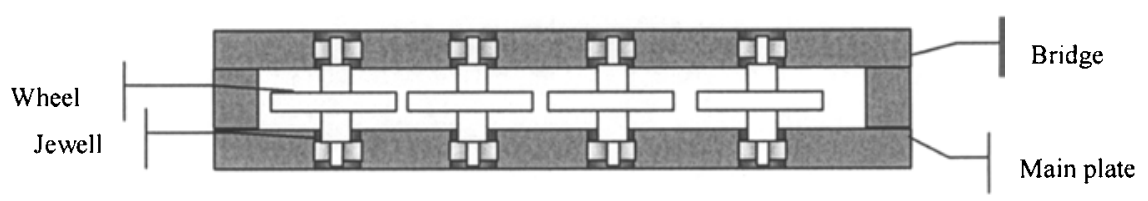

Fig. 1. Typical problem in watch making assembly 
To increase the yield (Manufacturing and assembly), different tolerancing methods are available, notably the worst case and statistical tolerancing methods. The worst-case method favours the assembly yield to the detriment of the manufacturing yield. Theoretically by this approach, if tolerances are respected, the assembly yield must be $100 \%$. Unfortunately though such tolerances are not compatible with the production and control means currently available. Statistical tolerancing introduced by Evans [9] through his state of the art could appear to be a solution. The statistical tolerancing method allows an important widening of the tolerances, but does not guarantee the conformity of the assembly. [2][3][13]. Graves[10] warns us against five fails of the statistical tolerancing limit and proposes some precautions at the time of statistical tolerancing design. But even with these precautions, statistical tolerancing remains dangerous.

Inertial tolerancing [4][5][6][7][8] proposes an alternative to the traditional methods, making it possible to obtain good yield at the same time in assembly and production. Tolerance does not define an interval but the maximum mean square deviation compared to the target (Equation 1).

$$
I=\sqrt{\delta^{2}+\sigma^{2}}
$$

I: Inertia

$\delta$ : Off-centring off the mean

$\sigma:$ Standard deviation

This new expression of the tolerance has many advantages. It makes it possible to increase variability in production while guaranteeing the functional clearance. This characteristic is particularly interesting in the complex case of the assembly of micromechanical parts. We propose to study the application of inertial tolerancing in the case of an assembly of a bridge on a main plate (Fig. 1). This assembly comprises $k$ wheels ( $k$ generally lying between 2 and 5), each wheel clearance implying a chain of $n$ components ( $n$ generally lying between 3 and 5).

\section{Assumptions:}

- The component count $c$ is identical for each wheel

- The functional clearance is identical for each wheel equal to $\pm t$

- In the case of a centred production, we wish to obtain a capability index $\mathrm{Cp}=$ 1 that is to say $(\sigma=t / 3)$

- Productions are normally distributed

- In the case of traditional tolerancing method, $C p$ is defined by Equation 2. In the case of inertial tolerancing method $C p$ is defined by Equation 3 


$$
\begin{aligned}
& C p=\frac{2 t}{6 \sigma} \\
& C p=\frac{I}{\sigma}
\end{aligned}
$$

In the case of a traditional tolerancing method, $C p k$ is defined by Equation 4 .

$$
C p k=\frac{\operatorname{Min}(\delta-L S L, U S L-\delta)}{3 . \sigma}=\frac{t-|\delta|}{3 . \sigma}
$$

In the case of an inertial tolerancing method, we defined $C p i$ by Equation 3.

$$
C p i=\frac{I_{0}}{I}
$$

$\mathrm{I}_{0}$ : Inertia tolerance

I: Inertia of the batch

\section{Calculation of inertial tolerance for each component}

Several approaches can be considered to calculate the inertial tolerance according to the assumptions of capability, and respect of the centering on the target. Two approaches were proposed which will be tested on this example.

1. Classical inertial tolerancing

2. Adjusted inertial tolerancing

We will compare these both approaches with the worst case and statistical tolerancing method for the yield of assembly and the variations authorizsed in production.

\section{Case \#1: Classical inertial tolerancing}

To calculate the component inertial tolerances $\left(I_{P}\right)$, the assembly distribution is considered centred, six standard deviations $( \pm 3 \sigma)$ contained in the functional tolerance interval $( \pm t)$. 


$$
I_{C}=\sigma_{C}=\frac{2 t}{6}
$$

With the precedent assumptions

$$
\begin{gathered}
I_{P}=I_{C} / \sqrt{n}=t / 3 \sqrt{n} \\
\mathrm{I}_{\mathrm{C}}: \text { Clearance Inertia } \\
\mathrm{I}_{\mathrm{P}}: \text { Part inertia }
\end{gathered}
$$

With the assumption of a capability $C p=2$ and $C p i=1$, the maximum decentring is equal to:

$$
\begin{aligned}
& \sigma_{P}=\frac{I_{P}}{2} \\
& \delta_{P}= \pm \sqrt{I_{P}^{2}-\sigma_{P}^{2}}= \pm \sqrt{\frac{3 I_{P}^{2}}{4}}= \pm \sqrt{\frac{3 I_{C}^{2}}{4 n}}
\end{aligned}
$$

The most unfavourable assembly corresponds to the situation where all decentrings are added. With the assumption of independence we can write:

$$
\begin{aligned}
& \delta_{C}=n \delta_{P} \\
& \sigma_{C}=\sqrt{n \sigma_{P}^{2}}=\sqrt{\frac{n I_{P}^{2}}{4}}=\sqrt{\frac{n I_{C}^{2}}{4 n}}=\frac{I_{C}}{2}=\frac{t}{6}
\end{aligned}
$$

With a tolerance of $\pm t$ on the clearance, the $\mathrm{z}$ index is calculated by the relation (10):

$$
z=\frac{t-n \sqrt{\frac{3 I_{C}^{2}}{4 n}}}{t / 6}=\frac{t\left(1-\sqrt{\frac{n}{12}}\right)}{t / 6}=6-\sqrt{3 n}
$$

The yield is calculated by $\Phi(z)$ the where $\Phi$ represent the standard normal cumulative distribution function. 


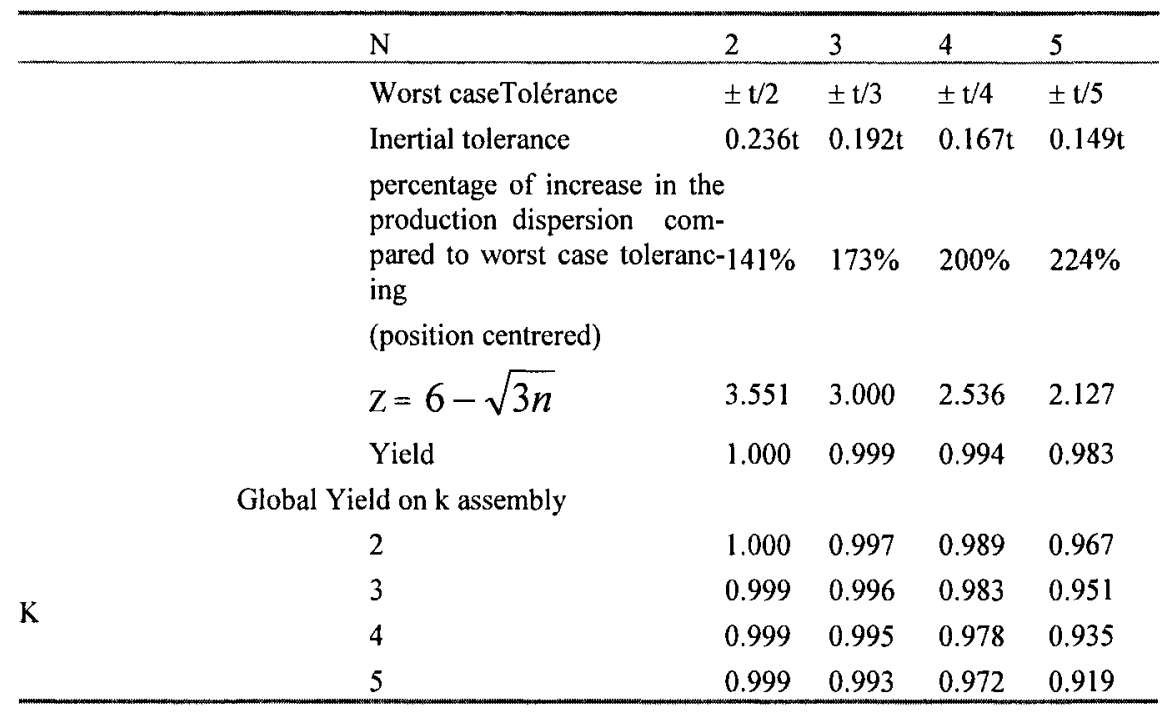

Table 1. Inertial Yield with tolerancing

\section{Case \#2: Statistical tolerancing}

The same table can be obtained for statistical tolerances under similar conditions: $\mathrm{Cp}=2, \mathrm{Cpk}=1$ :

$t_{P}=t / \sqrt{n}$

$$
\sigma_{P}=\frac{t_{P}}{6}=\frac{t}{6 \sqrt{n}}
$$

The maximum decentring is for $C p k=1$ :

$$
\delta_{P}=t_{P}-3 \sigma_{P}=\frac{t}{\sqrt{n}}-\frac{t}{2 \sqrt{n}}=\frac{t}{2 \sqrt{n}}
$$

The most unfavourable assembly corresponds to the situation where all decentrings are added [12]. With the assumption of independence we can write:

$\delta_{C}=n \delta_{P}=\frac{t \sqrt{n}}{2}$ 
$\sigma_{C}=\sqrt{n \sigma_{P}^{2}}=\frac{t}{6}$

The $z$ index is calculated by the relation (11):

$$
z=\frac{t-t \frac{\sqrt{n}}{2}}{t / 6}=6-3 \sqrt{n}
$$

\begin{tabular}{|c|c|c|c|c|c|}
\hline & $\mathrm{n}$ & 2 & 3 & 4 & 5 \\
\hline & Worst caseTolérance & $\pm \mathrm{t} / 2$ & $\pm t / 3$ & $\pm t / 4$ & $\pm \mathrm{t} / 5$ \\
\hline & statistical tolerance & $\pm 0.707 \mathrm{t}$ & $\pm 0.577 \mathrm{t}$ & $\pm 0.5 \mathrm{t}$ & $\pm 0.447 \mathrm{t}$ \\
\hline & \multicolumn{2}{|c|}{$\begin{array}{l}\text { percentage of increase in the } \\
\text { production dispersion com- } 141 \% \\
\text { pared to worst case toler- } \\
\text { ancing }\end{array}$} & $173 \%$ & $200 \%$ & $224 \%$ \\
\hline & $\mathrm{Z}=6-3 \sqrt{n}$ & 1.757 & 0.804 & 0.000 & -0.708 \\
\hline & Yield & 0.961 & 0.789 & 0.500 & 0.239 \\
\hline \multicolumn{6}{|c|}{ Global Yield on $\mathrm{k}$ assembly } \\
\hline & 2 & 0.923 & 0.623 & 0.250 & 0.057 \\
\hline \multirow{3}{*}{ K } & 3 & 0.886 & 0.492 & 0.125 & 0.014 \\
\hline & 4 & 0.851 & 0.388 & 0.063 & 0.003 \\
\hline & 5 & 0.818 & 0.306 & 0.031 & 0.001 \\
\hline
\end{tabular}

Table 2. Yield with statistical tolerancing

Table 2 shows the risks of the statistical tolerancing method. With a $C p=2$ on each component and $C p k=1$, as soon as there are 4 wheels in the mechanism the yield of the assembly can be close to zero although the individual yield of each component is 0.9973 .

In the case of inertial tolerancing the worst yield is equal to $0.919(k=5$ and $n=$ 4 ) for the same widening of the production spread in the centred case as statistical tolerances.

\section{Case \#3 - Adjusted inertial tolerancing}

Adragna [11] showed that it is possible to calculate inertia on each component in order to guarantee an yield of the final assembly.

Adragna proposed an inertial adjusted coefficient $\left(I_{C}\right)$ in order to guarantee that the assembly $C p k$ index will never be lower that a $C p k_{M i n}$ value. The $I_{C}$ coefficient is calculated by the simple following relation: 
$I_{C}=\sqrt{C p k_{M i n}^{2}+\frac{n}{9}}$

This $I_{C}$ coefficient has the same rule as the inflated coefficient for the traditional inflated statistical tolerancing:

$$
I_{P}=\frac{t}{6 \cdot I_{C} \cdot n^{1 / 2}}
$$

In our application we want to guarantee in all cases the assembly yield greater that $0.997\left(C p k_{\min }=1\right)$

$$
\begin{aligned}
& I_{C}=\sqrt{1+\frac{n}{9}} \\
& I_{P}=\frac{t}{3 \cdot \sqrt{n+\frac{n^{2}}{9}}}
\end{aligned}
$$

With the assumption of a capability $C p=2$ and $C p i=1$, the maximum decentring is equal to:

$$
\begin{gathered}
\sigma_{P}=\frac{I_{P}}{2}=\frac{t}{6 \sqrt{n+\frac{n^{2}}{9}}} \\
\delta_{P}= \pm \sqrt{I_{P}^{2}-\sigma_{P}^{2}}=\frac{ \pm t}{6} \frac{\sqrt{3}}{\sqrt{n+\frac{n^{2}}{9}}}
\end{gathered}
$$

The most unfavourable assembly corresponds to the situation where all decentrings are added. With the assumption of independence, we can write: $\delta_{C}=n \delta_{P}$ 


$$
\sigma_{C}=\sqrt{n \sigma_{P}^{2}}=\frac{t}{6 \cdot \sqrt{1+\frac{n}{9}}}
$$

With a tolerance of $\pm t$ on the clearance, the $\mathrm{z}$ index is calculated by the relation [10].

$$
z=\frac{t-\delta_{C}}{\sigma_{C}}=\frac{t-\frac{t}{6} \frac{n \sqrt{3}}{\sqrt{n+\frac{n^{2}}{9}}}}{6 \cdot \sqrt{1+\frac{n}{9}}}=6 \sqrt{1+\frac{n}{9}}-\sqrt{3 n}
$$

\begin{tabular}{|c|c|c|c|c|c|}
\hline & n & 2 & 3 & 4 & 5 \\
\hline & $\mathrm{I}_{\mathrm{C}}$ & 1.106 & 1.155 & 1.202 & 1.247 \\
\hline & $\mathrm{I}_{\mathbf{P}}$ & $0.2132 \mathrm{t}$ & $0.1667 \mathrm{t}$ & $0.1387 \mathrm{t}$ & $0.1195 \mathrm{t}$ \\
\hline & \multicolumn{2}{|c|}{$\begin{array}{l}\text { percentage of increase in the pro- } \\
\text { duction dispersion compared to } \\
\text { worst case tolerancing }\end{array}$} & $150 \%$ & $166 \%$ & $179 \%$ \\
\hline & \multicolumn{2}{|l|}{ (position centrered) } & & & \\
\hline & $z=6 \sqrt{1+\frac{n}{1}-\sqrt{3 n}}$ & 4.184 & 3.928 & 3.747 & 3.610 \\
\hline & Yield & 1.0000 & 1.0000 & 0.9999 & 0.9998 \\
\hline \multicolumn{6}{|c|}{$\begin{array}{l}\text { Global Yield on } k \text { as- } \\
\text { sembly }\end{array}$} \\
\hline & 2 & 1.000 & 1.000 & 1.000 & 1.000 \\
\hline \multirow{3}{*}{ k } & 3 & 1.000 & 1.000 & 1.000 & 1.000 \\
\hline & 4 & 1.000 & 1.000 & 1.000 & 0.999 \\
\hline & 5 & 1.000 & 1.000 & 1.000 & 0.999 \\
\hline
\end{tabular}

This relation gives the following outputs:

Table 3. Yield with adjusted inertial tolerancing

The yield is guarantees for each assembly. It is possible also to guarantee the global yield as we will detail it in the end of the article.

Is the situation of this article, yield is better than 0.997 . In fact the situation $C p=$ 2 and $C p i=l$ is not the worst case situation 


\section{Discussion}

The assembly of micromechanics components often leads to small yield of production and assembly because of the relative importance of the variability. In this context, it is important to choose a tolerancing method able to guarantee the functional specification for the product, but also to give the greatest possible variability for the production.

Worst case tolerances guaranteed the functional specification but is very restrictive for the production

Statistical tolerances gives freedom to the production but does not guaranteed a good assembly capability.

We show in this paper through generic example of assembly in watch making the great interest to use inertial tolerances. It guarantees at the same time good assembly capability while leaving the most possible freedom to the production.

\section{References}

1. S. Koelemeijer Chollet, F. Bourgeois, C. Wulliens, and J. Jacot. Cost modelling of microassembly. In Proceedings of the International Precision Assembly Seminar IPAS 2003, Bad Hofgastein, Austria, March 17-19, 2003.

2. Graves S. (2001) - Tolerance Analysis Tailored to your organization - Journal of Quality technology - Vol. 33, N³, 293-303, July 2001

3. K.W. Chase. Tolerance allocation methods for designers. ADCATS Report 99-6, Brigham YoungUniversity, 1999.

4. Pillet M., Inertial tolerancing in the case of assembled products, Recent advances in integrated design and manufacturing in mechanical engineering, No. ISBN 1-4020-1163-6, 2003, pp. 85-94,

5. Pillet M., Inertial Tolerancing, The Total Quality Management Magazine, Vol. 16, No. Issue 3 - Mai 2004, 2004, pp. 202-209,

6. F. Bourgeois, Y. L. de Meneses, S. Koelemeijer Chollet, and J. Jacot. Defining assembly specifications from product functional requirements using inertial tolerancing in precision assembly. In Proceedings of the IEEE International Symposium on Assembly and Task Planning, Montreal, July, 2005.

7. F. Bourgeois. Vers la maîtrise de la qualité des assemblages de précision. Thesis from Laboratory LPM1 Ecole Polytechnique de Lausanne(EPFL),Lausanne(Swiss), 2007

8. Adragna P.A. Tolérancement des Systèmes Assemblés, une approche par le Tolérancement Inertiel et Modal. Thesis from Laboratory Symme Polytech'Savoie, Annecy(France), december 2007.

9. Evans D.H., Statistical tolerancing the state of the art, newspaper of quality technology, vol. 7, No. 1, 1975

10. Graves S, Bisgaard S, Five ways statistical tolerancing can fail, and what to do about them, Quality Engineering, Vol.13,pp. 85-93.

11.Adragna P.A., Pillet M. Samper S., Formosa F., Guarantying a maximum of NonConformity Rate on the assembly resultant with a statistical tolerancing approach, Computer Aided Tolerancing (CAT) 2007. Erlangen, Germany.

12. Pillet.M, Inertial tolerancing, The TQM Magazine Volume 16 - Number 3 - 2004 - pp. 202-209

13. Nigam S.D and Turner JU Review of statistical approaches to tolerance analysis, computer aided Design Volume 27 Number 1 January 1995 pp 6-15 